\title{
Interventional Radiology for Whom
}

\author{
Yasuaki Arai ${ }^{1}$ (1)
}

Received: 24 October 2020/ Accepted: 29 October 2020/Published online: 20 January 2021

(C) Springer Science+Business Media, LLC, part of Springer Nature and the Cardiovascular and Interventional Radiological Society of Europe (CIRSE) 2021

There are approximately 200 countries on earth. How many of these countries can we name and locate? Publications on interventional radiology (IR) are made in various countries, yet, these include only less than 50 countries. Are other countries disease-free? No. Diseases that afflict people are everywhere around the world. Of course, research paper publication does not equal doing IR, but this gap means that there are many countries on this planet where IR is not being used towards helping people who suffer from illness. Most of the world's wealth is concentrated in only a handful of the developed countries [1], and IR is mostly done in these relatively economically rich countries. EBM (evidence-based medicine) is the basis of modern medicine and standard treatments are established through the rigorous clinical trials, often the best results of which are referred to as the Global standard. However, there are many people on the planet who cannot benefit from global standard treatments. We, interventional radiologists, must not forget this.

It goes without saying that medical care is greatly influenced by the economic condition of a country. It is difficult to provide medical care without a safe environment, a certain minimum supply of drugs, electricity, etc. Given that surgical treatment is performed in almost all countries even under difficult circumstances, IR should be used more, even with the handicap of lack of essential equipment. The biggest advantage of IR compared to other treatments is its "less invasiveness". Various studies aimed

Yasuaki Arai

arai-y3111@mvh.biglobe.ne.jp

1 Department of Diagnostic Radiology, National Cancer Center, 5-1-1, Tsukiji, Chuo-ku, Tokyo 104-0045, Japan at reducing invasiveness, increasing accuracy, reducing radiation exposure and so on, have been published in IR journals. However, the cost of these innovations has not been addressed. There are some papers published about costs, but the number is very limited because the costs are greatly influenced by the medical system of each country.

It is not easy to research and publish "cheaper IR"; for example, to reveal cheaper IR that has similar efficacy to existing standard treatments, a non-inferiority randomized controlled trial is needed [2], but this is extremely difficult in reality. It is also true that making the equipment essential for IR cheaper is not attractive to companies. Furthermore, in the increasingly strict medical safety and infection control trends, there is no conventional way to allow a slight decrease in efficacy and safety. Therefore, in order to develop "cheaper IR" for developing countries that are not economically wealthy, a different way of thinking may be necessary.

One key to solve the problem may be "technique". The paper, "FairEmbo concept for arterial embolizations: In vivo feasibility and safety with suture-based microparticles compared with microspheres" introduced the technique of "cutting the thread into small pieces" and showed the possibility that this technique can reduce the cost of IR [3]. In the current trend where most interventional radiologists hope to use expensive and high quality imaging equipment and devices, the attitude of conducting research and publishing papers from the viewpoint of "cheaper IR" is commendable $[4,5]$. We should also not forget that by using good technique, we may be able to accomplish similar quality IR with cheaper imaging equipment.

Technique is the greatest source of pride of interventional radiologists. The cost of technique is difficult to 
evaluate, but the least that could be said is that we have the right to make the decision.

Our IR should not be just for the wealthy. We should look to "cheaper IR" to make IR loved by everyone on the planet.

Funding None.

\section{Compliance with Ethical Standards}

Conflict of interest The author declares that there is no conflict of interest..

\section{References}

1. https://www.investopedia.com/insights/worlds-top-economies/
2. Snapinn SM. Noninferiority Trials Curr Control Trials Cardiovasc Med. 2000;1(1):19-21.

3. Di Bisceglie M, Hak J-F, Diop AD, et al. FairEmbo concept for arterial embolizations: in vivo feasibility and safety study with suture-based microparticles compared with microspheres. Cardiovasc Intervent Radiol. 2020. https://doi.org/10.1007/s00270-02002678-0.

4. Vidal V, Hak JF, Brige P, et al. In vivo feasibility of arterial embolization with permanent and absorbable suture: the FAIREmbo concept. Cardiovasc Intervent Radiol. 2019;42(8):1175-82.

5. Diop AD, Diop AN, Hak JF, et al. Hemostatic embolization of renal artery pseudoaneurysm using absorbable surgical suture (FairEmbo concept). Diagn Interv Imaging. 2020;101(11):757-8.

Publisher's Note Springer Nature remains neutral with regard to jurisdictional claims in published maps and institutional affiliations. 